\title{
The disconnection hypothesis
}

\author{
Karl J. Friston * \\ The Wellcome Department of Cognitive Neurology, Institute of Neurology, Queen Square, London, WCIN 3BG, UK
}

Received 3 June 1997; accepted 4 July 1997

\begin{abstract}
This article reviews the disconnection hypothesis of schizophrenia and presents a mechanistic account of how dysfunctional integration among neuronal systems might arise. This neurobiological account is based on the central role played by neuronal plasticity in shaping the connections and the ensuing dynamics that underlie brain function. The particular hypothesis put forward here is that the pathophysiology of schizophrenia is expressed at the level of modulation of associative changes in synaptic efficacy; specifically the modulation of plasticity in those brain systems responsible for emotional learning and memory, in the post-natal period. This modulation is mediated by ascending neurotransmitter systems that: (i) have been implicated in schizophrenia; and (ii) are known to be involved in consolidating synaptic connections during learning. The proposed pathophysiology would translate, in functional terms, into a disruption of the reinforcement of adaptive behaviour that is consistent with the disintegrative aspects of schizophrenic neuropsychology. (O) 1998 Elsevier Science B.V.
\end{abstract}

Keywords: Schizophrenia; Functional integration; Plasticity; Disconnection

\section{Introduction}

This article presents a disconnection hypothesis and discusses it in relation to underlying neurobiological mechanisms. Although disconnectionism provides a compelling framework for understanding schizophrenia it has to contend with two major problems: (i) There is little evidence for the anatomical interruption of connections in schizophrenia. (ii) Many deficits in schizophrenia can be explained by regionally specific abnormalities of function or physiology. Although these abnormalities could be framed in terms of abnormal connectivity, they do not in themselves constitute evidence

*Tel: 44171833 7456; Fax: 44171813 1445;

e-mail: k.friston@fil.ion.ucl.ac.uk for a pathology of integration. This paper is divided into three sections. In the first we address the two issues above. In the second section a mechanistic version of the disconnection hypothesis is presented. The third section reviews evidence in favour of schizophrenia as a functional disconnection syndrome.

\section{The nature of the disconnection hypothesis}

\subsection{What sort of disconnection syndrome?}

The disconnection hypothesis considered here states that schizophrenia can be understood in cognitive terms, and in terms of pathophysiology, as a failure of proper functional integration within 
the brain. Functional integration refers to the integration of functionally specialized systems (i.e., populations of neurons, cortical areas and subareas), that is required for adaptive sensorimotor integration and cognition. Functional integration is mediated by the influence that the dynamics or activity of one neuronal system exerts over another and is therefore determined by the connections between them. The pattern of connections is, in turn, a function of epigenetic, activity- and experience-dependent plasticity that underlies ontology and the post-natal remodelling of synaptic connections. This article suggests that the pathophysiology of schizophrenia involves the modulation or facilitation of long-term changes in connection strength. This is distinct from an abnormality of plasticity per se and focuses on the aberrant regulation of where and when in the brain plasticity occurs. This dynamic regulation can be attributed, in part, to the ascending modulatory neurotransmitter systems. This hypothesis is mechanistic. It is not aetiological in that it only specifies the processes that are implicated in a proximate sense.

The notion that psychosis can be explained by a pathology of extrinsic connections (cortico-cortical and cortico-subcortical connections that constitute white matter tracts) can be attributed to Wernicke. This hypothesis is anatomical and localises the pathology to interruptions of, or abnormalities in, the anatomical connections themselves. This is not the sort of disconnection syndrome proposed for schizophrenia. In schizophrenia, the disconnection is explicitly functional, not anatomical (Friston and Frith, 1995). More precisely the disconnection is in terms of effective connectivity (Gerstein and Perkel, 1969) as opposed to anatomical connectivity. The abnormal interactions between neuronal populations will clearly have infrastructural correlates, but these are likely to be expressed at the level of synaptic specialisations, cellular morphology and cytoarchitectonics. Any misconception that the disconnection hypothesis pertains to the extrinsic connections themselves is understandable given that evidence, cited in support of the disconnection hypothesis, refers explicitly to interruptions of cortico-cortical connections (e.g., psychotic symptoms in metachromatic leukodystrophy; Hyde et al., 1992). However, it is important to remember that this sort of evidence is being used as a 'lesion deficit' model of a functional disconnection. These observations are used to make the point that psychotic symptoms emerge when functional interactions are disrupted by anatomical lesions, not that anatomical lesions are aetiologically important. The concern about the lack of evidence for abnormal white matter in schizophrenia could be replaced by the critical question: 'How many of the neurochemical and cytoarchitectonic abnormalities in schizophrenia are a consequence of putative disruptions of experience-dependent plasticity that is normally responsible for modelling the cellular and sub-cellular infrastructure of neuronal circuits?'.

\subsection{Dysfunctional integration or dysfunctional specialisation}

It is worthwhile considering the disconnection hypothesis in relation to other formulations. The alternative to the disconnection hypothesis would suggest that schizophrenia can be explained by regionally specific pathophysiology in one or more neuronal systems; in other words, impaired functional specialization as opposed to dysfunctional integration. There is an important distinction here between the pathological interaction of two cortical areas and the otherwise normal interaction of two pathological areas. What follows is an attempt to show why this is fundamental.

The distinction between a regional and an interactional pathology begs a related question 'Which has ontological primacy, functional specialization or functional integration?'. From the perspective of the disconnection hypothesis functional integration supervenes: the primary pathology is supposed to be enacted at the level of neuronal interactions and, in particular, the induction and maintenance of a proper pattern of synaptic connections. A failure to establish this pattern will necessarily result in abnormalities of functional specialisation. The disconnection hypothesis therefore subsumes both abnormalities of functional integration and specialisation but the latter (i.e., abnormalities of local processing, neuronal dynamics and infrastructure) are secondary. The distinction between a regionally specific insult to 
the brain and aberrant interactions is fundamental. A regionally specific pathology, such as stroke or a tumour, is a sufficient explanation for the particular cognitive or sensorimotor deficits of some patients. With the exception of psychomotor poverty syndromes these lesion deficit models are not generally useful in schizophrenia. In terms of neuropsychology, many of the experiential symptoms and positive signs of schizophrenia can only be explained by considering one cognitive process in relation to another: for example, hallucinations can be construed as a mis-attribution of internally generated speech to an outside agency (Frith, 1992). This speaks to a failure to integrate the attribution of agency and inner speech. In other words, the symptoms and signs of schizophrenia do not generally represent a single deficit but can be seen as resulting from the abnormal integration of two or more processes. In a similar vein the disconnection hypothesis suggests that the neuronal dynamics underlying these symptoms are not due to a single regionally specific pathophysiology but are expressed when two or more regions interact. This is not to say that the regions involved will not show region-specific abnormalities but that these abnormalities are epiphenomena of a more pervasive problem. The alternative perspective, where dysfunctional integration is secondary to a regionally specific insult, is a good model for other organic brain syndromes. For example, a middle cerebral artery infarction, resulting in aphasia, can be comprehensively understood in terms of a specific insult to the cortical areas functionally specialised for language. Although the damaged areas will not interact properly with other frontal and temporal regions, the abnormal interactions are secondary.

The distinction between dysfunctional integration and dysfunctional regional specialisation is neither specious nor a question of perspective. It may be thought, given that the dynamics (spatiotemporal patterns of neural activity) in a cortical area are a function of the intrinsic connectivity (local connections, confined to the grey matter) and input from other areas, that one could not meaningfully divorce functional specialisation from the integration mediated by these inputs. However, the disconnection hypothesis is not simply a matter of emphasis on the inputs, as opposed to the changes in the dynamics that they evoke. It has fundamental implications for empirical observations of brain function: for example, consider the patterns of neural activity in the temporal lobe of schizophrenics. Suppose that these are abnormal relative to control subjects. The explanation for this abnormality may be of two sorts. Firstly, it could reflect pathology that is entirely intrinsic to the temporal regions, rendering them abnormally responsive to inputs from elsewhere or, secondly, they could result from abnormal interactions with the prefrontal cortices. These two explanations would lead to very different predictions and inferences. The first explanation would predict that temporal responses would be abnormal irrespective of the events or tasks used to elicit them. The disconnection explanation, however, would predict that abnormal temporal responses would only be seen in the context of tasks that involved interactions within a distributed system that necessarily included the prefrontal cortex and temporal regions. It is conceivable that both temporal and prefrontal responses would appear quite normal in situations that did not typically rely on prefronto-temporal interactions. In other words, the abnormal temporal responses are only revealed or expressed in relation to prefrontal dynamics. It should be noted, however, that simply demonstrating abnormal integration between the prefrontal and temporal cortices does not represent sufficient evidence for a disconnection. Both regions should be demonstrated to have viable responses to inputs from other areas (e.g., the superior temporal region should still respond to afferent input from the periauditory or parietal regions).

\section{The disconnection hypothesis}

\subsection{Connections and plasticity}

The key assumption here is that the pathophysiology of schizophrenia is expressed in terms of abnormal connections. Because synaptic connections are in a continual state of flux, this implies an abnormality of time-dependent changes in con- 
nectivity i.e., abnormal plasticity. The disconnection hypothesis is therefore, implicitly, a dysplastic hypothesis. The aim of this section is identify the particular form of dysplasia that might underlie schizophrenia (see also Haracz, 1985).

To understand functional disconnection, in its broadest sense, one has to appreciate the diversity of mechanisms that are responsible for establishing the complicated and functionally adaptive pattern of connections in the normal brain. These result from an interplay between genetic, epigenetic and activity or experience-dependent mechanisms. The emphasis in utero is clearly on epigenetic mechanisms; such as the interaction between the spatiotemporal topography of the developing cortical sheet, cell migration, gene expression and the mediating role of gene-gene interactions and gene products such as cell adhesion molecules (CAMs). Following birth, the broad schema of connections are progressively refined and remodelled with a greater emphasis on activity- and use-dependent plasticity. These changes endure into adulthood with ongoing reorganisation and experiencedependent plasticity that subserves behavioural adaption and learning throughout life. In summary, there are two basic determinants of connectivity in the brain. (i) Epigenetic: reflecting the interactions between the molecular biology of gene expression, cell migration and the slow spatiotemporal dynamics of morphogenesis and ontology in the developing brain. (ii) Activity-dependent modelling of the pattern and strength of synaptic connections. Activity-dependent plasticity can be divided into (a) plasticity that depends on interactions between the epigenetically determined patterns of connectivity and neuronal activity intrinsic to the developing nervous system; and (b) experience or use-dependent changes in synaptic efficacy that are elicited by interactions with the environment. The latter are associated with learning and memory that subserve adaptive or compensatory changes in emotional, cognitive or sensorimotor integration. This plasticity may be transient (e.g., short-term potentiation or depression) or enduring (e.g., long-term potentiation). It may or may not be associated with synaptic remodelling and infrastructural changes in cell processes (e.g., terminal arbors or dendritic spines; Rotshenker,
1988). It may rely purely on local dynamics or it may be influenced by other (modulatory) neurotransmitter systems (e.g. Bear and Singer, 1986; Kasamatsu, 1991; McGaugh, 1992).

\subsection{The plastic processes implicated in schizophrenia}

A first step in identifying the processes that are affected by schizophrenic pathophysiology is to note that symptoms, reminiscent of those in schizophrenia, can be produced by psychomimetic drugs (e.g. Allen and Young, 1978). This immediately tells one that the locus of abnormality is likely to be at the modulation of synaptic efficacy (either short- or long-term) in the post-developmental period. Secondly, the fact that schizophrenia is expressed symptomatically in adulthood points to an abnormal modulation of experience-dependent plasticity, as distinct from the induction and maintenance of synaptic connections through epigenetic mechanisms or indeed activity-dependent plasticity in utero: for example, there is no evidence to suggest that the ocular dominance columns in the striate cortex of schizophrenics are abnormal. This does not preclude neurodevelopmental explanations for schizophrenia (see Weinberger, 1987; and Murray and Lewis, 1987), but suggests that the endpoint of any putative aetiology affects biological processes that are only expressed in the postdevelopmental period. Thirdly, schizophrenia is a brain syndrome that has a degree of specificity in terms of the functional, anatomical and neurotransmitter systems that have been implicated: given the ubiquitous nature of short- and longterm potentiation (and depression) of synaptic efficacy throughout the brain, schizophrenia is unlikely to be associated with abnormal short or long-term plasticity per se but could be associated with the abnormal control or modulation of plasticity; where this control is exerted by systems with a circumscribed anatomy and neurochemistry: from the perspective of the disconnection hypothesis it is of no surprise that the neurotransmitter systems implicated in schizophrenia are exactly those that are responsible for modulating shortand long-term changes in synaptic plasticity. The role of the ascending classical neuromodulatory 
transmitters systems such as dopamine (DA) and acetylcholine (ACh) in modulating short-term changes in efficacy at a synaptic and cell assembly level is well known (e.g. Mesulam, 1990; GoldmanRakic et al., 1989; Mantz et al., 1988; Sawaguchi and Goldman-Rakic, 1991; Williams and Goldman-Rakic, 1995): However, these ascending systems also have a crucial role in modulating long-term associative plasticity that may be even more important in elaborating and maintaining an adaptive pattern of connections. We now consider this role in some detail.

\subsection{Modulation of long-term changes in synaptic efficacy}

There is considerable evidence to suggest monoaminergic (DA, norepinephrine, NE, and serotonin, 5HT) and cholinergic (ACh) neurotransmission facilitates either the induction or maintenance of long-term changes in synaptic strength. The three most compelling lines of evidence are modulation of (i) experience-dependent changes in synaptic efficacy; (ii) behavioural plasticity; and (iii) experimentally induced long-term potentiation. One neurodevelopmental example is the role of $\mathrm{ACh}$ in facilitating experience-dependent organization of connections in striate cortex: induction of ocular-dominance shift in cats, and its recovery following monocular deprivation depend on the integrity of NE and/or ACh neurotransmission (Bear and Singer, 1986). ACh and NE have both been shown to modulate experiencedependent modification of synaptic connectivity, possibly in a synergistic way (Brocher et al., 1992). Further evidence implicating $\mathrm{ACh}$ in the modulation of plasticity comes from the electrophysiological correlates of learning. Metherate and Weinberger (1989) have demonstrated conditioned stimulus-specific modification of frequency-receptive fields in cat auditory cortex during conditioning. Pairing of exogenous acetylcholine and a single tone resulted in a similar shift, with maximal change at the frequency paired with acetylcholine. These findings suggest that $\mathrm{ACh}$ is necessary for, and can modulate, associative plasticity in a learning-related context. In short, the ascending neurotransmitter systems can exert a regulatory control over the induction and maintenance of long-term changes in connections that are associated with learning and memory. From the perspective of theoretical neurobiology (in particular, neuronal selection and reinforcement learning) this modulatory effect has profound implications for the way that adaptive connections can be established. Any related pathophysiology (e.g., schizophrenia) can usefully be understood from this perspective.

\subsection{The theoretical importance of modulating associative plasticity}

The existence of neurotransmitter systems that can facilitate associative plasticity, has profound implications for the development and maintenance of adaptive connections. The basic idea has two levels: first, if such neurotransmitter systems exist then any events that lead to a discharge of activity in these systems will consolidate the pattern of synaptic connections extant at that time. This provides a powerful mechanism to select connections that mediate adaptive sensorimotor integration. Clearly, for this selection to work only events that are 'adaptive' or have 'value' should excite modulatory discharges of this sort (i.e., the outcome of any sensorimotor integration should be adaptive or valuable for the phenotype). The ability of events to elicit activity in ascending modulatory systems will be determined by the inputs (afferents) to the cells of origin of these systems (e.g., the VTA for DA or the nucleus basalis for ACh). These afferents therefore determine what is valuable (e.g. a reversal of hypoglycaemia). How that valuable state is achieved depends on selective consolidation of adaptive responses to salient stimuli (e.g., conditioned responses to appetitive stimuli) mediated by discharges in the ascending systems. The key notion here is that the inputs to modulatory systems can be specified genetically, therein providing an essential link between evolutionary selection (what is adaptive about a response to a cue) and neuronal selection (how that response is acquired or selectively consolidated in relation to other competing responses). By embedding neuronal selection in evolutionary selection in this way the elaboration of adaptive behaviour (i.e., learning) can be seen 
as a simple emergent phenomenon. The second level considers the fact that the inputs to the modulatory systems are themselves subject to the same modulation-dependent selection. A simple consequence of this is that cues or salient stimuli can acquire 'value' if, and only if, they are associated with adaptive responses. These stimuli can themselves now consolidate or reinforce antecedent behavioral responses leading, by recursion, to a succession of adaptive stimulus-contingent responses. This sequence must, by the nature of its construction, arrive at a genetically specified and valuable endpoint.

The above analysis predicts, and relies upon, transient discharges in ascending modulatory systems that are associated with the processing of salient stimuli that elicit adaptive responses. An experimental model of this situation is operant conditioning, particularly higher-order reinforcement schedules: experimental evidence for reinforcement-specific responses in these systems is clear-cut: for example, the experiments of Ljungberg et al. (1992) have demonstrated phasic discharges in the cells of origin of the dopaminergic system that are selective for reinforcing stimuli. Other models include intracranial self-stimulation and studies of emotional learning in animals (see Cador et al., 1989; Metherate and Weinberger, 1989; McGaugh, 1992; White and Milner, 1992). A full exposition of these ideas will be found in Friston et al. (1994) discussed in the context of the theory of neuronal group selection (Edelman, 1993) and reinforcement learning.

\subsection{Abnormal modulation in schizophrenia}

What would follow from an improper functioning of these modulatory mechanisms in the light of the theoretical analysis above? As an example, consider the ascending mesocortico-limbic dopaminergic system. The distribution of the terminal fields of these ascending neurons shows a regional specificity with a predilection for the prefrontal cortices, and related limbic and paralimbic structures (Oades and Halliday, 1987). Suppose now that the associative plasticity in these structures was modulated or facilitated in an aberrant fashion. Functionally this would correspond to an uncoupling between the sensorimotor and cognitive contingencies encountered in the environment and the reinforcement experienced. The disruptive effect on adaptive behaviour and underlying patterns of connections is obvious. There would be a greatly reduced facility to engage in tasks that involved high-order conditioning and a concomitant difficulty with developing coherent strategies of adaptive behaviour. If one conjectures that most of the 'reinforcement' in adult life derives from our interactions with other people, then the integration of positive or negative feedback from our transactions with others into our repertoire of social responses would become impossible. In short, there would be a failure of social functioning and deterioration; as previously reinforced behaviours were 'overwritten' by anomalous facilitation of plasticity. An important aspect of the theoretical perspective is the recursive consolidation of a temporal succession of neuronal dynamics, each reinforcing its antecedent. A failure of this 'chaining' can be related, in at least a speculative way, to the disorganized and incoherent language produced by some schizophrenics and the perseverative and 'autistic' aspects of their behaviour.

\subsection{Explanatory power}

It is proposed that schizophrenia is a functional disconnection syndrome mediated by some form of dysplasia. We have identified the consolidation or modulation of associative changes in synaptic efficacy (as opposed to the changes themselves) as the process that is most likely to be affected and have focused on the ascending neurotransmitter systems in this role. An important aspect of the disconnection hypothesis is that this sort of pathophysiology will only be expressed in those systems that: (i) support plastic change; and (ii) are subject to modulation by the neurotransmitter system or systems in question. This allows for entirely normal activity-dependent plasticity in systems that are not subject to such modulation or in those that have already passed through a critical period of plastic organization (e.g., the visual cortex). One might anticipate that by early adulthood only a portion of the brain will be amenable to reinforcement-dependent changes in synaptic efficacy; 
namely, those systems implicated in [emotional] learning and memory. A pathophysiology restricted to these systems is consistent with many aspects of schizophrenia: (i) the cardinal deficit would be a failure to develop or maintain coherent and adaptive patterns of reinforced behaviour and is consistent with many of the disintegrative aspects of schizophrenic neuropsychology. (ii) The abnormality can be framed in terms of experience or activity-dependent plasticity in systems that are only functionally expressed in the developed brain. This allows for the fact that psychomimetic drugs can induce psychotic symptoms and that schizophrenia is primarily a disorder of adulthood. (iii) The abnormality shows a degree of anatomical and neurochemical specificity; the medial temporal lobe (amygdala hippocampus, parahippocampal gyrus and related cortex) and the prefrontal cortex are directly implicated by virtue of their role in learning and memory and their high plastic potential (Haracz, 1985). The fact that these structures have been repeatedly implicated in schizophrenia by functional and structural imaging, cytoarchitectonic and histochemical studies (e.g., Benes et al., 1986; Deakin et al., 1989; Bogerts et al., 1991) is entirely consistent with the current formulation. (iv) It directly posits a mechanistic role for the ascending modulatory neurotransmitter systems that have been implicated in schizophrenia from both a pharmacological perspective (e.g., Dolan et al., 1995) and in terms of animal models (e.g., Sawaguchi and Goldman-Rakic, 1991). (v) It explicitly brings together molecular biology (consolidation of synaptic efficacies) and behaviour (experience-dependent learning) in a mechanistic way. Bridging between molecular and systemslevel descriptions is important, both from a phenomenological point of view (e.g., the stressdiathesis model of schizophrenia) and in terms of directed research.

\subsection{Predictions of the disconnection hypothesis}

The experimental predictions that ensue are fairly straightforward. (i) Schizophrenics should evidence abnormal functional integration, indexed by abnormal effective connectivity in neuroimaging, and coherence among electrical and biomag- netic signals. These markers of functional disconnection should be seen in, and only in, systems that have a high plastic potential, or equivalently systems that are involved in learning and memory. (ii) Schizophrenics should be impaired on tasks that emphasis temporal succession, reinforcement and new learning, such as highorder reinforcement schedules and reversal learning. (iii) There should be a demonstrable difference between normal subjects and schizophrenics, in terms of how synaptic connections are consolidated during learning; and (iv) more specifically how drugs that manipulate the function of ascending neurotransmitter systems will modulate associative plasticity. (v) Discharge rates in ascending neurotransmitter systems will be abnormal in relation to the presentation of stimuli in high-order reinforcement schedules. This is not to say that the pathophysiology affects these systems directly but that the connections to these systems, that mediate their responses, are likely to be abnormal. It is unclear how this could be measured in humans at present; however recent developments in dynamic displacement studies of receptor-specific radioligands with positron emission tomography may represent a possible way forward. (vi) A more speculative experimental prediction is that cytoarchitectonic and biochemical changes seen in schizophrenia may be emulated in animal models where the presentation of stimuli and reinforcers in operant paradigms has been disrupted by experimental design. One could think of many more examples but these serve to illustrate the usefulness of a simple and mechanistic hypothesis.

\section{Evidence for the disconnection hypothesis}

This section focuses on evidence for schizophrenia as a functional disconnection syndrome. It should be noted that the notion of disconnection derives not only from neurophysiological data but also from cognitive models of schizophrenia dating back, perhaps, to Bleuler (1911). A more recent formulation of psychological 'disintegration' is the idea that some experiential symptoms of schizophrenia can be explained by a failure to integrate the intention to act with the perceptual registration 
of the consequences of that action (Feinberg, 1978; Frith, 1992). At a neurobiological level this integrative abnormality might correspond to a failure to integrate signals from the (intentional) prefrontal regions and the (perceptual) temporal cortices. The notion that schizophrenia represents a disintegration or fractionation of the psyche is as old as its name introduced by Bleuler (1911) to convey a 'splitting' of mental faculties. The lines of evidence reviewed below relate to the neurophysiological basis of this mentalistic splitting.

\subsection{Anatomical disconnections}

Although the proposal here is that schizophrenia is a functional disconnection syndrome, conditions that involve anatomical disconnections may provide good lesion-deficit models. It is interesting to note that Wernicke proposed that psychosis may result from disruption of the 'organs of connection' (white matter fibre tracts) at about the same time that Bleuler introduced the term schizophrenia. However, the evidence for white matter pathology in schizophrenia is not remarkable. Having said this, white matter disorders such as metachromatic leukodystrophy do provide compelling models of schizophrenia, particularly where the pathophysiology may involve an anatomical deafferentation of the prefrontal cortex (Hyde et al., 1992). See also Bruton et al. (1994). The sequelae of a functional disconnection syndrome could of course be expressed anatomically even at a macroscopic level. MRI studies of callosal anatomy suggest a significant reduction in callosal area in schizophrenia although this is not a consistent finding (Woodruff et al., 1995). There are a number of cytoarchitectonic studies that could be interpreted in terms of abnormal cell migration and the formation of aberrant connections in neurodevelopment (e.g. Jacob and Beckman, 1986).

\subsection{Neurochemical disconnections}

In the same sense that functional disconnections may be modelled by, or have, anatomical correlates, neurochemical studies may also provide models. For example phencyclidine (PCP) is a psychomimetic drug that induces schizophrenia- like symptoms (Allen and Young, 1978). PCP is a potent inhibitor of $N$-methyl-D-aspartate (NMDA) glutamate receptors. Glutamate receptors are ubiquitous in the brain and glutamate neurotransmission is the mainstay for the excitatory cortico-cortical interactions that integrate different brain areas. PCP psychosis could then be construed as a transient chemical disconnection syndrome affecting these connections. While a primary pathology of glutamate receptors is not necessarily an explanation for functional disconnection, it is likely that a disruption of corticocortical interactions at any level will be reflected in abnormalities of glutamate function. Schizophrenics have been shown to have abnormal markers of glutamate function both ex vivo (Deakin et al., 1989; Sherman et al., 1991) and in vivo (Pettegrew et al., 1991).

\subsection{Functional disconnections and neuroimaging}

Functional connectivity in neuroimaging is defined as a correlation between remote neurophysiological events and effective connectivity as the influence that one neuronal system or population exerts over another (Friston et al., 1993). Measures of functional connectivity are closely related to coherence in multi-channel electrophysiological recordings or correlations in multiunit electrode recordings (e.g., Gerstein and Perkel, 1969; Aertsen and Preissl, 1991). In positron emission tomography and functional MRI neuroimaging studies, functional connectivity is usually assessed on the basis of the correlations in a time series of brain scans. Our own analyses, of the interactions between prefrontal and temporal cortices, suggest that schizophrenics have an abnormal pattern of functional connectivity involving both an absence of correlations between prefrontal and superior temporal regions and the presence of abnormal positive correlations between prefrontal and more posterior middle temporal cortex (Friston et al., 1996). These observations are provisional but replicate.

\subsection{Theoretical neurobiology}

Theoretical neurobiology and, in particular, computational neurobiology can be useful in help- 
ing to explain the relationship between emergent phenomena in mathematical models and those seen in real brains. For example, connectionist models have been used to explore the link between cognitive impairment and dopamine neurotransmission. Cohen and Servan-Schreiber (1992) have studied attention and language processing in relation to dopaminergic modulation of the sensitivity of prefrontal units to input. Hoffman and McGlashan (1993) have related the dynamics of fully interconnected Hopfield-type networks to symptoms of schizophrenia by looking at the effect of successively reducing the connections between the interacting units. Our own work using synthetic neuronal models has looked at the relationship between the amount of connectivity among neuronal populations and measures of complexity using nonlinear dynamics (the correlation dimension): in brief, we see a progressive increase in the correlation dimension when connectivity is reduced (Friston, 1996). This is consistent with a functional disconnection in schizophrenia in the sense that at least some studies show that the correlation dimension is elevated in schizophrenia (e.g. Koukkou et al., 1993). Again it should be noted that a functional disconnection syndrome does not necessarily imply a reduced number of anatomical connections but simply an abnormal influence of one neuronal population over another. Aberrant functional interactions could, conceivably, be associated with hypertrophic or exuberant connections (see David, 1994).

\subsection{Neural plasticity in schizophrenia}

Neural plasticity refers to the capacity of the nervous system to exhibit structural and functional adaptations to impinging stimuli. Long-termpotentiation (LTP) is considered to be one of the best models of plasticity that might underlie learning. The association between temporal lobe epilepsy and schizophrenic symptoms (e.g., Ounsted and Lindsay, 1981) can be related to plastic mechanisms through a predisposition to kindling phenomena (see Cain, 1989 for a comparison of LTP and kindling). Mapping studies indicate that LTP and kindling phenomena are especially demonstrable in regions that have been implicated in schizo- phrenia. Hippocampal pathways show the strongest and most enduring LTP effects, but LTP is also seen throughout the limbic forebrain. The regional specificity of plastic potential has also been addressed by looking for the expression of gene products associated with plastic remodelling of neuronal processes. See Haracz (1985) for a review of the experimental evidence relating neural plasticity and schizophrenia.

There is considerable histopathological evidence for abnormal plasticity in schizophrenia including: (i) decreased hippocampal expression of the embryonic form of the neural cell adhesion molecule (NCAM) in schizophrenia. This form of NCAM has been proposed to be related to synaptic rearrangement and plasticity (Barbeau et al., 1995). (ii) Selective displacement of interstitial white matter neurons in the frontal lobes of schizophrenics that may reflect an alteration in the migration of subplate neurons or in the pattern of programmed cell death. Both could be associated with abnormal neural circuitry and connectivity (Akbarian et al., 1996). Electron microscopy of synaptic ultrastructure in regions associated with the dopaminergic system has revealed changes that are consistent with anomalous plasticity: namely, anomalous axonal sprouting with an increase in axospinous, and decrease in axodendritic, synapses in schizophrenia (Uranova, 1996).

\section{Conclusion}

This article has reviewed the disconnection hypothesis of schizophrenia and has presented a mechanistic account of how dysfunctional integration among neuronal systems might arise. The particular hypothesis put forward is that the pathophysiology of schizophrenia is expressed at the level of modulation of associative changes in synaptic efficacy; specifically the consolidation of associative plasticity in those brain systems responsible for emotional learning and memory. This modulation is mediated by ascending neurotransmitter systems that: (i) have been implicated in schizophrenia; and (ii) are known to be involved in consolidating synaptic plasticity. By reference to theoretical neurobiology this pathophysiology can 
be understood as disrupting the reinforcement of adaptive behaviour in a way that is consistent with the disintegrative aspects of schizophrenic neuropsychology.

\section{Acknowledgment}

I thank my colleagues at the Wellcome Department of Cognitive Neurology for invaluable discussion, particularly Chris Frith. KJF is supported by the Wellcome Trust.

\section{References}

Aertsen, A., Preissl, H., 1991. Dynamics of activity and connectivity in physiological neuronal networks. In: Schuster, H.G. (Ed.), Non Linear Dynamics and Neuronal Networks. VCH, New York, NY, pp. 281-302.

Akbarian, S., Kim, J.J., Potkin, S.G., Hetrick, W.P., Bunney, W.E., Jones, E.G., 1996. Maldistribution of interstitial neurons in prefrontal white matter of the brains of schizophrenic patients. Arch. Gen. Psychiatry 53, 425-436.

Allen, R.W., Young, S.J., 1978. Phencyclidine-induced psychosis. Am. J. Psych. 135, 1081-1084.

Barbeau, D., Liang, J.J., Robitalille, Y., Quirion, R., Srivastava, L.K. 1995. Decreased expression of the embryonic form of the neural cell adhesion molecule in schizo. phrenic brains. Proc. Natl. Acad. Sci. USA, 92, 2785-2789.

Bear, M.F., Singer, W., 1986. Modulation of visual cortical plasticity by acetylcholine and noradrenaline. Nature 320 , 172-176.

Benes, F.M., Davidson, J., Bird, E.D., 1986. Quantitative cytoarchitectural studies of the cerebral cortex of schizophrenics. Arch. Gen. Psychiatry 43, 31-35.

Bleuler, E., 1911. Dementia Praecox or the Group of Schizophrenias. English Translation 1961. International Universities Press, New York.

Bogerts, B., Ashtari, M., Degreef, G.J., Alvir, J., Bilder, R.M., Leiberman, J.A., 1991. Reduced temporal limbic structure volumes on magnetic resonance images in first episode schizophrenia. Psychiatric Res Neuroimaging 35, 1-13.

Brocher, S., Artola, A., Singer, W., 1992. Agonists of cholinergic and noradrenergic receptors facilitate synergistically the induction of long-term potentiation in slices of rat visual cortex. Brain Res. 573, 27-36

Bruton, C.J., Stevens, J.R., Frith, C.D., 1994. Epilepsy, psychosis and schizophrenia: clinical and neuropathologic correlations. Neurology 44, 34-42.

Cador, M., Robbins, T.W., Everitt, B.J., 1989. Involvement of the amygdala in stimulus-reward associations: interaction with the ventral striatum. Neuroscience $30,77-86$.

Cain, D.P., 1989. Long-term potentiation and kindling: how similar are the mechanisms. Trends in Neurosciences 12 $6-10$.

Cohen, J.D., Servan-Schreiber, D., 1992. Context, cortex and dopamine: a connectionist approach to behavior and biology in schizophrenia. Psychol. Rev. 99, 45-77.

David, A.S., 1994. Schizophrenia and the corpus callosum: developmental, structural and functional relationships. Behav. Brain Res. 64, 203-211.

Deakin, J.F.W., Slater, P., Simpson, M.D.C., Gilchrist, A.C., Skan, W.J., Royston, M.C., Reynolds, G.P., Cross, A.J., 1989. Frontal cortical and left temporal glutaminergic dysfunction in schizophrenia. J. Neurochem. 52, 1781-1786.

Dolan, R.J., Fletcher, P., Frith, C.D., Friston, K.J., Frackowiak, R.S.J., Grasby, P.M., 1995. Dopaminergic modulation of impaired cognitive activation in the anterior cingulate cortex in schizophrenia. Nature 378, 180-182.

Edelman, G.M., 1993. Neural Darwinism: selection and reentrant signalling in higher brain function. Neuron 10, 115-125

Feinberg, I., 1978. Efference copy and corollary discharge implications for thinking and its disorders. Schizophr. Bull 4, 636-640.

Friston, K.J., Frith, C.D., Liddle, P.F., Frackowiak, R.S.J., 1993. Functional connectivity: the principal component analysis of large (PET) data sets. J. Cereb. Blood Flow Metab. 13, 5-14.

Friston, K.J., Tononi, G., Reeke, G.H., Sporns, O., Edelman, G.E., 1994. Value-dependent selection in the brain: simulation in a synthetic neural model. Neuroscience 39, 229-243.

Friston, K.J., Frith, C.D., 1995. Schizophrenia: a disconnection syndrome? Clin. Neurosci 3, 89-97.

Friston, K.J., 1996. Theoretical neurobiology and schizophrenia. Br. Med. Bull. 52, 644-655.

Friston, K.J., Herold, S., Fletcher, P., Silbersweig, D., Cahill, C., Dolan, R.J., Liddle, P.F., Frackowiak, R.S.J., Frith, C.D., 1996. Abnormal fronto-temporal interactions in schizophrenia In: Watson, S.J. (Ed.), Biology of Schizophrenia and Affective Disease. ARNMD Series, Vol. 73, pp. 421-429.

Frith, C.D., 1992. The Cognitive Neuropsychology of Schizophrenia. Lawrence Erlbaum, Sussex, UK.

Gerstein, G.L., Perkel, D.H., 1969. Simultaneously recorded trains of action potentials: analysis and functional interpretation. Science $164,828-830$.

Goldman-Rakic, P.S., Leranth, C., Williams, S.M., Mons, N., Geffard, M., 1989. Dopamine synaptic complex with pyramidal neurons in primate cerebral cortex. Proc. Natl. Acad. Sci. USA 86, 9015-9019.

Haracz, J.L., 1985. Neural plasticity in schizophrenia. Schizophr. Bull. 2, 191-229.

Hoffman, R.E., McGlashan, T.H., 1993. Parallel distributed processing and the emergence of schizophrenia symptoms. Schizophr. Bull. 19, 119-140.

Hyde, T.M., Ziegler, J.C., Weinberger, D.R., 1992. Psychiatric disturbances in metachromatic leukodystrophy-insights into the neurobiology of psychosis. Arch. Neurol. 49, 401-406.

Jacob, H., Beckman, H., 1986. Prenatal development distur- 
bances in the limbic allocortex in schizophrenics. $\mathbf{J}$. Neural Trans. 65, 303-326.

Kasamatsu, T., 1991. Adrenergic regulation of visuocortical plasticity: a role of the locus coeruleus system. Prog. Brain Res. 88, 599--616.

Koukkou, M., Lehmann, D., Wackermann, J., Dvorak, I., Henggeler, B., 1993. Dimensional complexity of EEG brain mechanisms in untreated schizophrenia. Biol. Psychiatry 33, 397-407.

Ljungberg, T., Apicella, P., Schultz, W., 1992. Responses of monkey dopamine neurones during learning of behavioral reactions. J. Neurophysiol. 67, 145-163.

Mantz, J., Milla, C., Glowinski, J., Thierry, A.M., 1988. Differential effects of ascending neurons containing dopamine and noradrenaline in the control of spontaneous activity and of evoked responses in the rat prefrontal cortex. Neuroscience 27, 517-526.

McGaugh, J. (1992) Neuromodulatory systems and the regulation of memory storage. In: Squire, L., Butters, N. (Eds.), Neuropsychology of Memory. Guildford Press, New York, NY, pp. 386-401.

Mesulam, M.M., 1990. Large scale neurocognitive networks and distributed processing for attention language and memory. Ann Neurol. 28, 597-613.

Metherate, R., Weinberger, N.M., 1989. Acetylcholine produces stimulus-specific receptive field alterations in cat auditory system. Brain Res. 480, 372-377.

Murray, R.M., Lewis, S.R., 1987. Is schizophrenia a developmental disorder? Br. Med. J.ournal. 295, 681-682.

Oades, R.D., Halliday, G.M., 1987. Ventral tegmental (A10) system: Neurobiology. 1. Anatomy and connectivity. Brain Res. Rev. 12, 117-165.
Ounsted, C., Lindsay, J., 1981. In: Reynolds, E.H., Trimble, M.R. (Eds.), Epilepsy and Psychiatry. Churchill Livingstone, Edinburgh.

Pettegrew, J.W., Keshavan, M.S., Panchalingam, K., Strychor, S., Kaplan, D.B., Tretta, M.G., Allan, M., Alterations in brain high-energy phosphate and membrane phospholipid metabolism in first episode, drug-naive schizophrenics: a pilot study of the dorsal prefrontal cortex by phosphorus 31 nuclear magnetic resonance spectroscopy. 1991. Arch. Gen. Psych. 48, 563-568.

Rotshenker, S., 1988. Multiple nodes and sites for the induction of axonal growth. Trends Neurosci. 11, 363-366.

Sawaguchi, T., Goldman-Rakic, P.S., 1991. D1 dopamine receptors in prefrontal cortex: involvement in working memory. Science 251, 947-950.

Sherman, A.D., Davidson, A.T., Baruah, S., Hegwood, T.S., Waziri, R., 1991. Evidence of glutaminergic deficiency in schizophrenia. Neurosci. Lett. 122, 77-90.

Uranova, N.A., 1996. Brain synaptic plasticity in schizophrenia. Vestn. Ross. Akad. Med. Nauk. 4, 23-29.

Weinberger, D.R., 1987. Implications of normal brain development for the pathogenesis of schizophrenia. Arch. Gen. Psychiatry 44, 660-669.

White, N.M., Milner, P.M., 1992. The psychobiology of reinforcers. Ann. Rev. Psychol. 43, 443-471.

Williams, G.V., Goldman-Rakic, P.S., 1995. Modulation of memory fields by dopamine D1 receptors in prefrontal cortex. Nature 376, 572-575.

Woodruff, P.W., McManus, I.C., David, A.S., 1995. Metaanalysis of corpus callosum size in schizophrenia. J. Neurol. Neurosurg. Psychiat. 58, 457-461. 